NASA Technical Memorandum 104469

AIAA-91-1758

\title{
3-D Navier-Stokes Analysis of Crossing, Glancing Shocks/Turbulent Boundary Layer Interactions
}

D.R. Reddy

- Lewis Research Center

Cleveland, Ohio

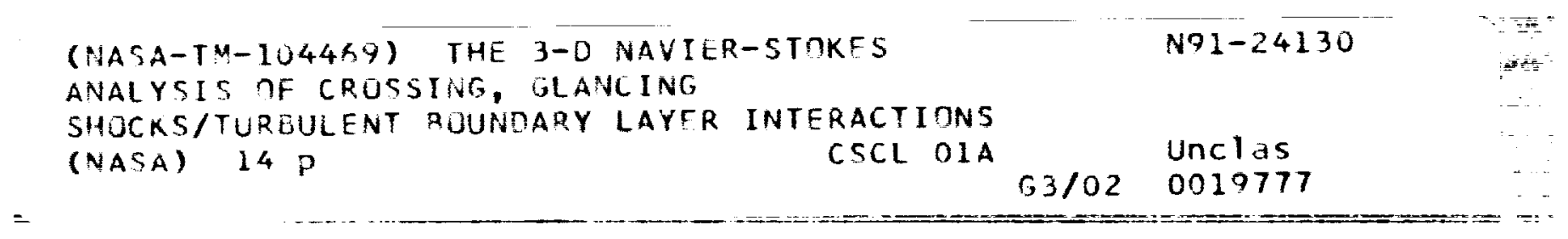

Prepared for the

22nd Fluid Dynamics, Plasma Dynamics and Lasers Conference sponsored by the American Institute of Aeronautics and Astronautics Honolulu, Hawaii, June 24-26, 1991 


\title{
3-D NAVIER-STOKES ANALYSIS OF CROSSING, GLANCING
}

\section{SHOCKS/TURBULENT BOUNDARY LAYER INTERACTIONS}

\author{
D. R. Reddy* \\ National Aeronautics and Space Administration \\ Lewis Research Center \\ Cleveland, Ohio 44135
}

\begin{abstract}
Three-dimensional viscous flow analysis is performed for a configuration where two crossing and glancing shocks interact with a turbulent boundary layer. A time marching 3-D full Navier-Stokes code, called PARC3D, is used to compute the flow field and the solution is compared to the experimental data obtained at the NASA Lewis Research Center $1 \mathrm{ft}$. $X \quad 1 \mathrm{ft}$. supersonic wind tunnel facility. The study is carried out as part of the continuing code assessment program in support of the Generic Hypersonic research at NASA Lewis. Detailed comparisons of static pressure fields and oil flow patterns is made with the corresponding solution on the wall containing the shock/boundary layer interaction in an effort to validate the code for hypersonic inlet applications.
\end{abstract}

\section{Introduction}

The shock wave/boundary-layer interaction phenomenon is a common occurrence in many of the important propulsion components such as supersonic inlets, nozzles and supersonic combustors. The strong pressure gradients, secondary flows and possible flow separations associated with these interactions can have significant effect on the flow through the individual components and consequently affect the overall performance of the engine. Therefore, a thorough understanding of these phenomena is essential for an efficient design of the propulsion components in the supersonic flow regime. To simulate these complex flows with a reasonable accuracy, a full 3-D NavierStokes code with a fairly reliable turbulence model is required. Therefore, it is important that the code selected for computation of these flows be thoroughly assessed using experimental data to verify the code's capability to correctly simulate the flow features mentioned above. The object of this study is to assess the numerical code, used to compute such flows, using experimental data obtained for a

"Chief, Computational Fluid Dynamics Branch. Member AIAA. 
configuration where two crossing and glancing shocks interact with a turbulent boundary layer. The experiment was carried out at the NASA Lewis Research Center $1 \mathrm{ft} X \quad 1 \mathrm{ft}$ supersonic wind tunnel facility.

The numerical code is a time marching full Navier-Stokes code, called PARC3D', which solves the full three dimensional Reynolds averaged NavierStokes equations in strong conservation form using Beam and Warming approximate factorization. The code was originally developed as AIR3D by Pulliam and Steger $^{2}$, and Pulliam later added the Jameson $^{4}$ artificial dissipation and called the code ARC3D. Cooper ${ }^{9}$ adapted the code for internal flow in propulsion application and named the code PARC3D. The PARC3D code uses central differencing on a generalized curvilinear coordinate system with implicit and explicit second and fourth order artificial dissipation. To simplify the solution of the block pentadiagonal system of discretized equations, the block implicit operators are diagonalized by decomposing the flux Jacobians, resulting in a scalar pentadiagonal system. The loss of time accuracy from the diagonalization does not affect the spatial accuracy of the steady state solution. ${ }^{3}$ The turbulence model used in the code for this study is the Baldwin-Lomax mode $7^{5}$.

The PARC3D code has been verified previously with experimental data for 3-D supersonic and 2-D hypersonic flow configurations having flow features similar to those of the present study $6,7,8$. These studies demonstrated the capability of PARC $3 D$ to simulate with reasonable accuracy the shock/boundarylayer interaction phenomenon typically present in the supersonic inlet configurations. This study is a continuation of the code validation process being carried out at NASA Lewis Research Center for computations of flows through supersonic/hypersonic propulsion components in support of the Generic Hypersonic research program.

\section{Experimental Configuration}

The crossing shock/boundary-layer experiment was configured by using two shock generator plates that span the tunnel test section. The $20.3 \mathrm{~cm}$ long shock generators arranged symmetrically produce oblique shocks which cross each other on the centerline. The interaction of these shocks with the naturally occurring incoming flow boundary layer on the tunnel wall defines the experiment. This interaction is shown schematically in Fig. 1. The incoming boundary layer was observed to be uniform and fully turbulent. The thickness of the boundary layer varied between 3.0 to $3.5 \mathrm{~cm}$ depending on the inflow Mach number.

The experiment was run at different shock generator angles of 4, 6, 10 and 12 degrees for a Mach number varying from 2.5 to 4.0. However, all the angles were not run at each Mach number because the model would unstart and produce a normal shock ahead of the shock generator for some combinations of Mach number and angles; for example for Mach 2.5, a shock generator angle of $9 \mathrm{deg}$. is the $1 \mathrm{imiting}$ value. The experimental data include surface static pressure measurements and oil flow visualization on the tunnel 
wall. Complete details of the experimental configuration and instrumentation used in the experiment can be found in reference 9 .

\section{Computations}

The computations are performed on the Numerical Aerodynamic Simulation's Cray-2 computer, located at NASA Ames Research Center, using a grid size of 151 $X 71 \times 71$. The computational grid showing one each of the streamwise and cross planes is shown in Fig. 2. To resolve the viscous layers, the grid lines are clustered in regions close to the walls using hyperbolic tangent functions such that the first grid line away from the wall is located at a $\mathrm{y}^{+}$of approximately 2.0. Since the configuration is symmetric in both directions in the cross plane, only a quarter of the full flow field is computed with symmetry imposed in the vertical as well as the transverse direction along the planes passing through the centerline of the wind tunnel. The flow field at the inflow boundary, which is a plane just ahead of the shock generators, is held fixed. This flow field consists of an incoming 2-D turbulent boundary layer, on the tunnel wall, which is matched with that of the experiment. Since most of the flow in this $\mathrm{plane}$ including a large part of the boundary layer is supersonic, the fixed boundary condition at this plane does not violate any physics of the flow. At the outflow boundary, once again since the flow is mostly supersonic, the flow variables are extrapolated from inside. The outflow boundary is located several $\mathrm{planes}$ beyond the shock generator trailing edges. The distance between the trailing edge and the boundary is about $25 \%$ of the length of the shock generator.

A nonreflective boundary condition, using a simple Mach wave extrapolation, is applied on that portion of the computational boundary extending from the trailing edge of the shock generator to the outflow boundary plane. This boundary condition allows the expansion waves from the trailing edge of the shock generator to pass through the free boundary. A no-slip condition is applied on the solid walls. In the present computations the turbulence model is modified such that when a reversed flow region is encontered, the outer layer model chooses the maximum of the two eddy viscosity values computed by BaldwinLomax formulation and P. D. Thomas ${ }^{10}$ model. In addition, based on the study of Deiwert ${ }^{11}$, the inner layer is replaced with the outer model which extends all the way upto the wall in this region. In the absence of reversed flow regular Baldwin-Lomax two-layer formulation is used.

\section{Results and Discussion}

Solutions are obtained for the cases corresponding to the experimental runs of Mach numbers 3.5 and 4.0. Only two shock generator angles, 6 and 10 deg., are considered for computation because the experimental flow visualization data are available for only these two angles for a Mach number of 3.5. For the Mach 3.5 case both 6 and $10 \mathrm{deg}$. configurations are computed, whereas for the Mach 4.0 case on Ty $6 \mathrm{deg}$. geometry is analyzed. 
Figure $3 a$ and $3 b$ show the static pressure contours on the tunnel wall for the case of 6 deg. shock generator angle and Mach numbers of 3.5 and 4.0 compared with the experimental data. The comparison shows a good agreement between the experimental data and the solution. The corresponding static pressure contours for the 10 deg. shock generator angle and Mach 3.5 case are shown in Fig. $3 c$ in comparison with the experimental data. The comparison shows that the prediction in general agrees fairly well with the experimental data.

Static pressure distributions along the centerline of the tunnel wall for 6 deg. shock generator angle and Mach numbers of 3.5 and 4.0 are presented in Fig. $4 a$ and $4 b$ along with the corresponding experimental data. The comparison shows a very good agreement between the solution and the experimental data. The centerline pressure distribution for the 10 deg. case and Mach 3.5 is compared with the experimental data in Fig. 4c. Except for a slight diagreement upstream of the plateau region of the curve, the solution agrees fairly well with the experimental data. This slight disgreement is due to the discrepency in the location and extent of the predicted reversed flow region compared to that of the experiment. The difference between the predicted and the experimental reversed flow region and its effect on the pressure distribution are discussed later in this section.

Figures $5 a$ and $5 b$ show the particle traces close to the tunnel wall from the prediction and the oil flow pattern from the experimental data respectively for the $6 \mathrm{deg}$. and Mach 3.5 configuration. The particle traces are restricted to the $X Y-p l a n e$ to approximately duplicate the oil flow pattern on the wall. Since these pictures are supposed to show the flow features qualitatively, the agreement between the solution and the data is good.

The particle traces and oil flow pattern corresponding to the $10 \mathrm{deg}$. case are shown in Fig. $6 \mathrm{a}$ and $6 \mathrm{~b}$. Both the solution and experimental data show a reversed flow region in the middle of the tunnel wall. However, in the prediction, the region is located slightly upstream and extends over a larger area compared to that in the experimental data. In the experimental study ${ }^{9}$ it was pointed out that the presence of oil in the flow could change the character of the flow and so the extent and the location of the reversed flow region could be different from that of the flow without the $0 i 1$. Due to the difference in the location and extent of the reversed flow region, the predicted pressure distribution shows the plateau, a characteristic of the reversed flow, slightly upstream and larger in extent compared to the experimental value (see Fig. 4c). The discrepency between the prediction and the experimental data in the reversed flow region could also be partly attributed to the approximations in the turbulence model, used in the code, which is an algebraic eddy viscosity model. To improve the accuracy of predictions in the reversed flow regions it might be necessary to use higher order turbulence models which are currently being investigated at NASA Lewis Research center under the code 
validation efforts in the Generic Hypersonic reasearch program.

\section{Summary}

3-D viscous flow solutions have been obtained for a configuration where two crossing and glancing shocks interact with a turbulent boundary layer in order to assess the PARC3D code for hypersonic inlet applications. The solutions for different shock generator angles and Mach numbers have been compared to the experimental data which include static pressure flelds and oil flow patterns on the wall which contained the interaction. The comparison shows that the PARC $3 D$ code is capable of predicting the complex flow features, typically present in supersonic and hypersonic inlets, with a reasonably good accuracy. To further improve the accuracy in computing the reversed flow regions, application of higher order turbulence models needs to be examined in the future code validation studies.

\section{Acknowledgement}

Part of this research was carried out under contract NAS3-25366 supported by NASA Lewis Research Center when the author was employed at the Lewis Research Center Group of Sverdrup Technology, Inc.

\section{References}

1 Cooper, G. K., Jordan, J. L., and Phares, W. J., "Analysis Tool for Application to Ground Testing of Highly Underexpanded Nozzles", AIAA $87-2015,1987$.

2 Pulliam, T. H., and Steger, J. L., "Implicit Finite-Difference
Simulations of Three Dimensional Compressible Flow", AIAA Journal, Vo1. 18, February 1980, pp. 159167 .

Pulliam, T. H., "Euler and Thin Layer Navier-Stokes Code: ARC2D, ARC3D", Notes for Computational Fluid Dynamics User's Workshop, The University of Tennessee Space Institute, Tullahoma, Tennessee, March 12-16, 1984.

Jameson, A., Schmidt, W., and Turkel, E., "Numerical Solutions of the Euler Equations by Finite Volume Method Using Runge-Kutta Time Stepping Schemes", AIAA 81$1259,1981$.

5 Baldwin, B., and Lomax, H., "Thin Layer Approximtion and Algebraic Model for Separated Flows, "AIAA Paper 78-257, Jan. 1978.

Reddy, D. R., and Harloff, G. J., "Three Dimensional Viscous Flow Computations and High Area Ratio Nozzles for Hypersonic Propulsion", Journal of Propulsion and Power, Vol. 7 , No. 1, January-February 1991, pp. 84-89.

Reddy, D. R., Smith, G. E., Liou, M.-F., and Benson, T. J., "Three Dimensional Viscous Analys is of a Hypersonic Inlet", AIAA paper 890004, January 1989.

Reddy, D. R., Benson, T. J., and Weir, L. S., "Comparison of 3-D Viscous Flow Computations of Mach 5 Inlet with Experimental Data", AIAA paper 90-0600, January 1990.

Hingst, W. R. and Williams, K. E., "Interaction of Two Glancing, Crossing Shock Waves with a Turbulent Boundary Layer at Various Mach Numbers," NASA TM 103740, 1991. 
10 Thomas, P. D., "Numerical Method for Predicting Flow Characteristics and Performance of Nonaxisymmetric Nozzles - Theory, " Langley Research Center, NASA CR 3147, September 1979.

11 Deiwert, G. S., "Computation of Separated Transonic Turbulent Flows," AIAA Journal, Vol. 14, No. 6, June 1976, pp. 735-740.

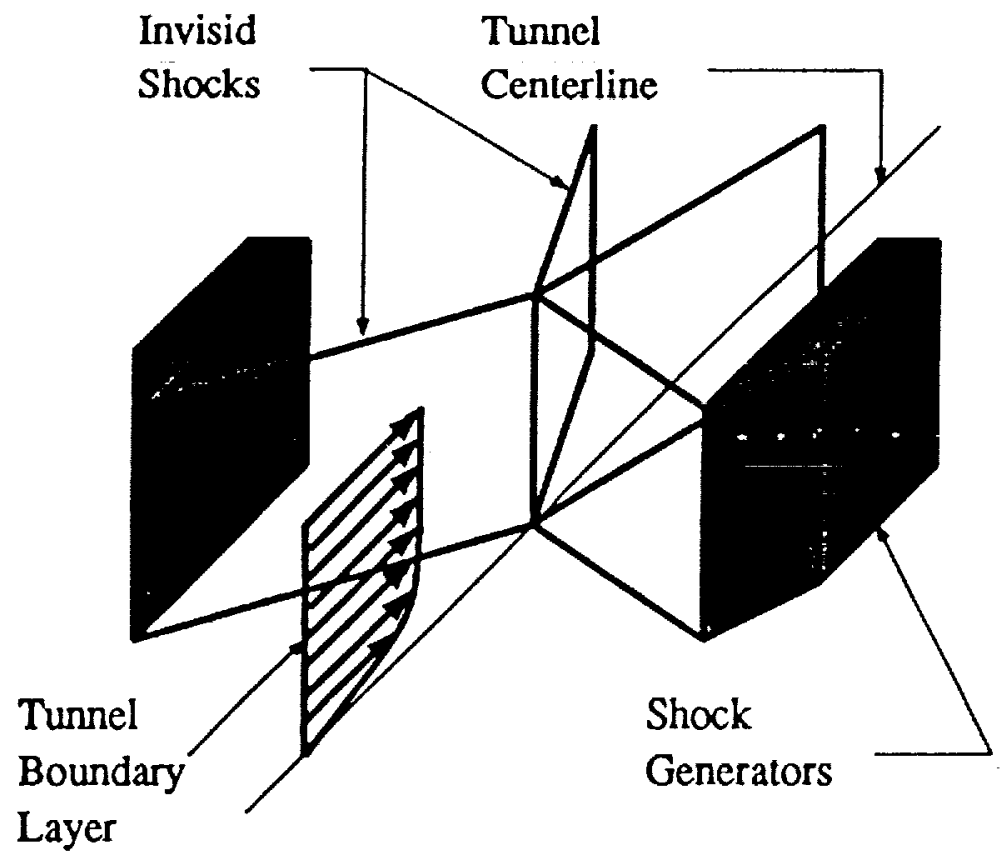

Fig. 1 Experimental Configuration 


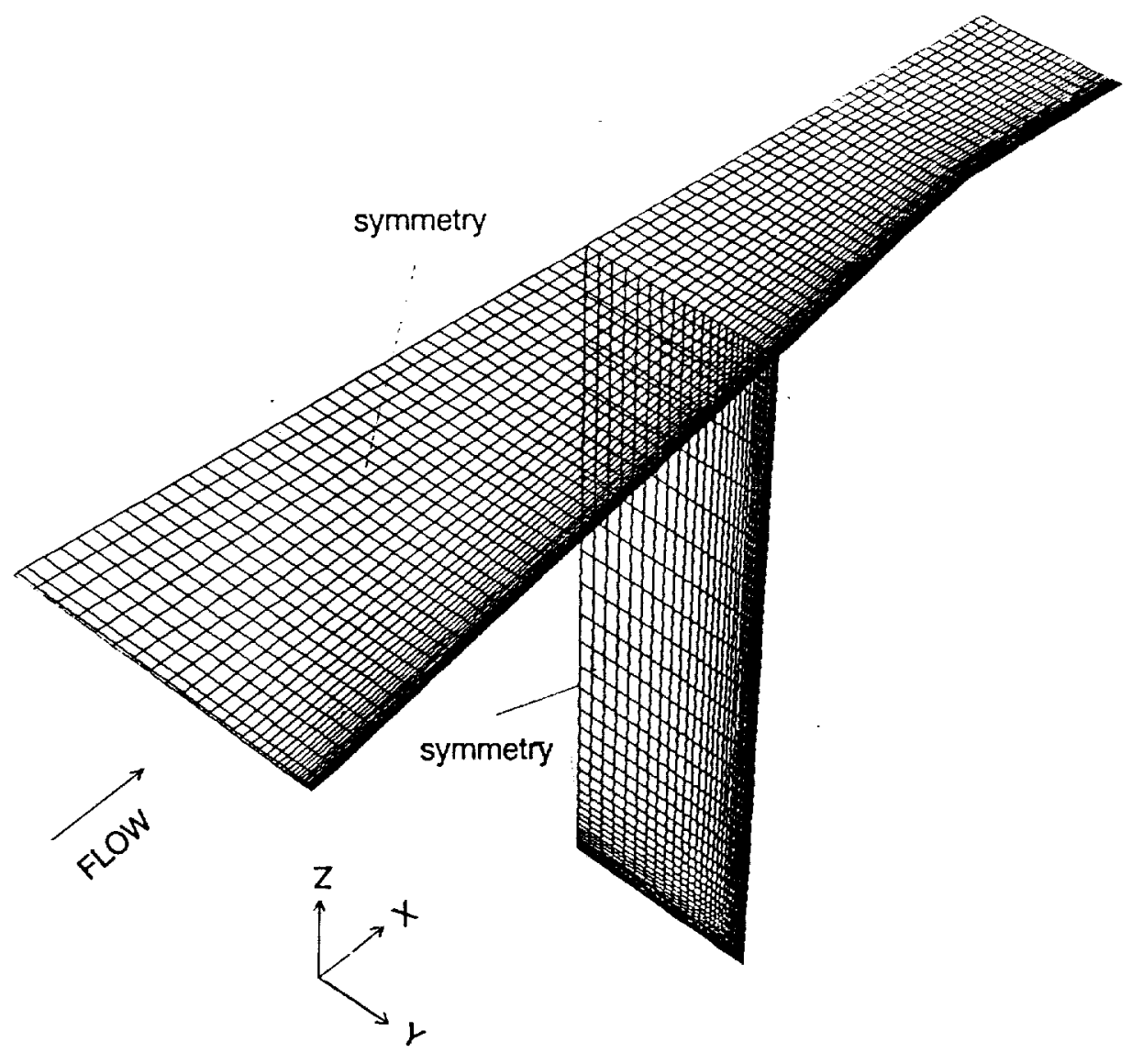

Fig. 2 Computational Grid 


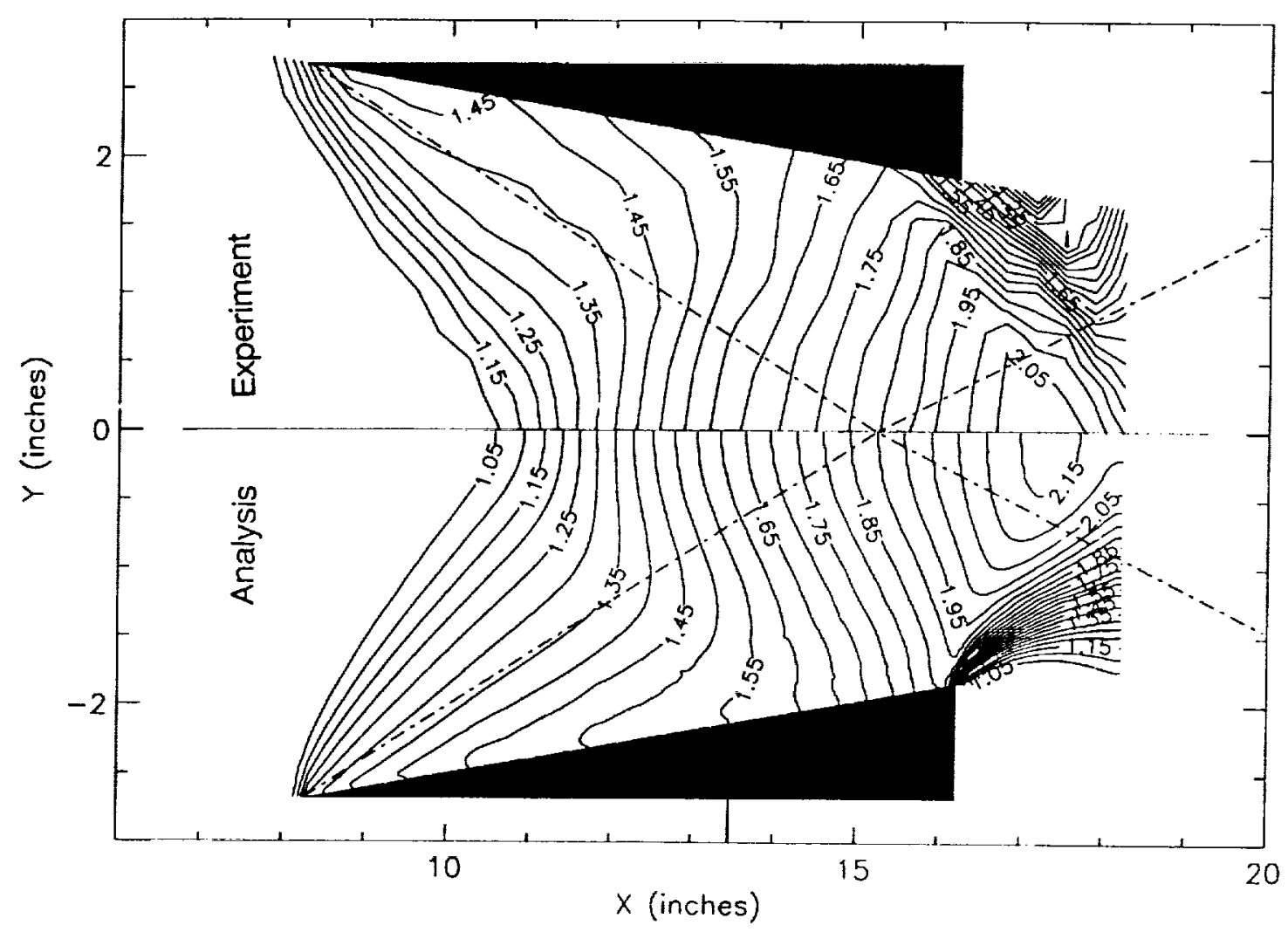

Fig. 3a Pressure Contours for 6 deg. Mach 3.5

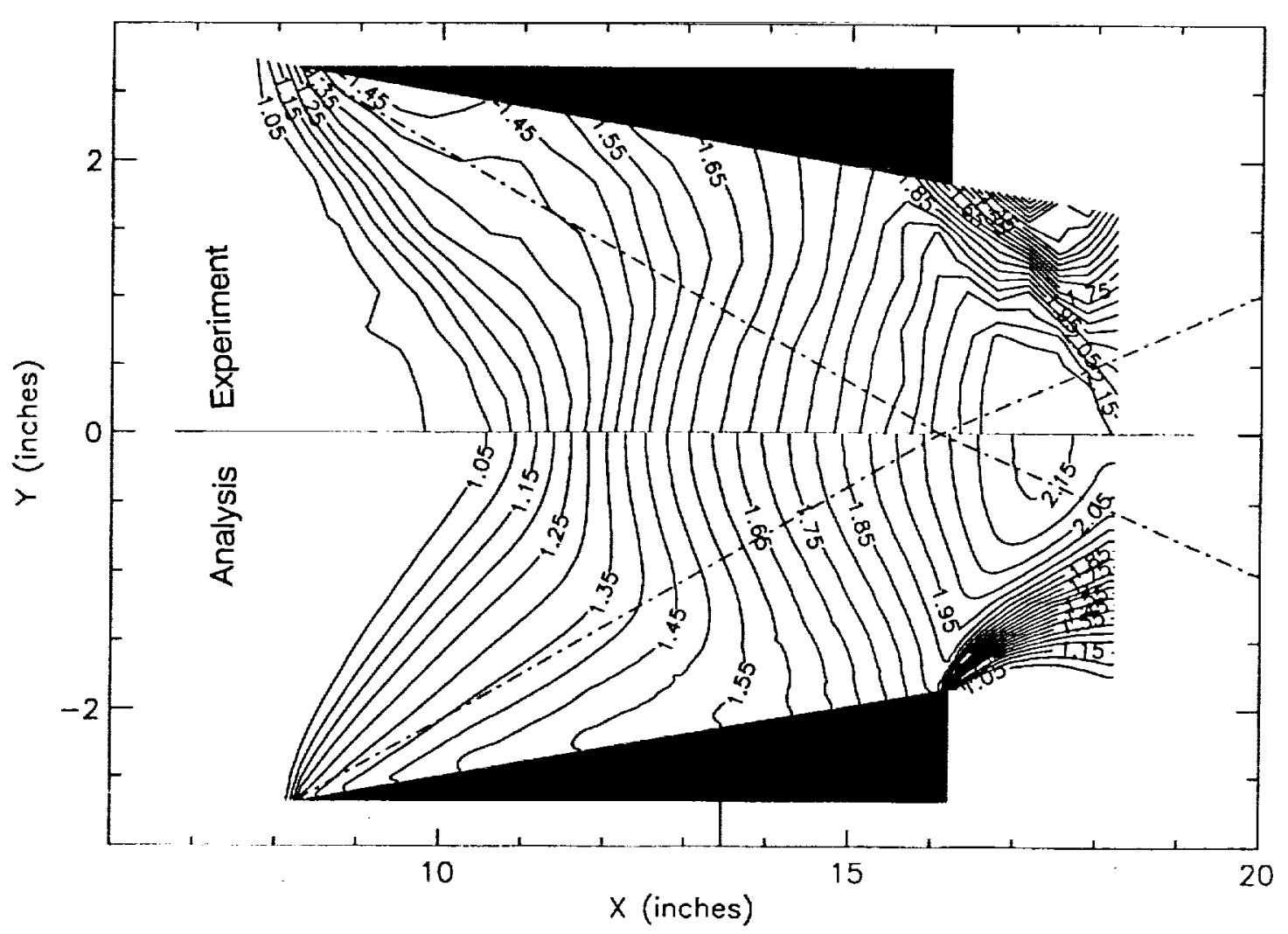

Fig. 3b Pressure Contours for 6 deg. Mach 4.0 


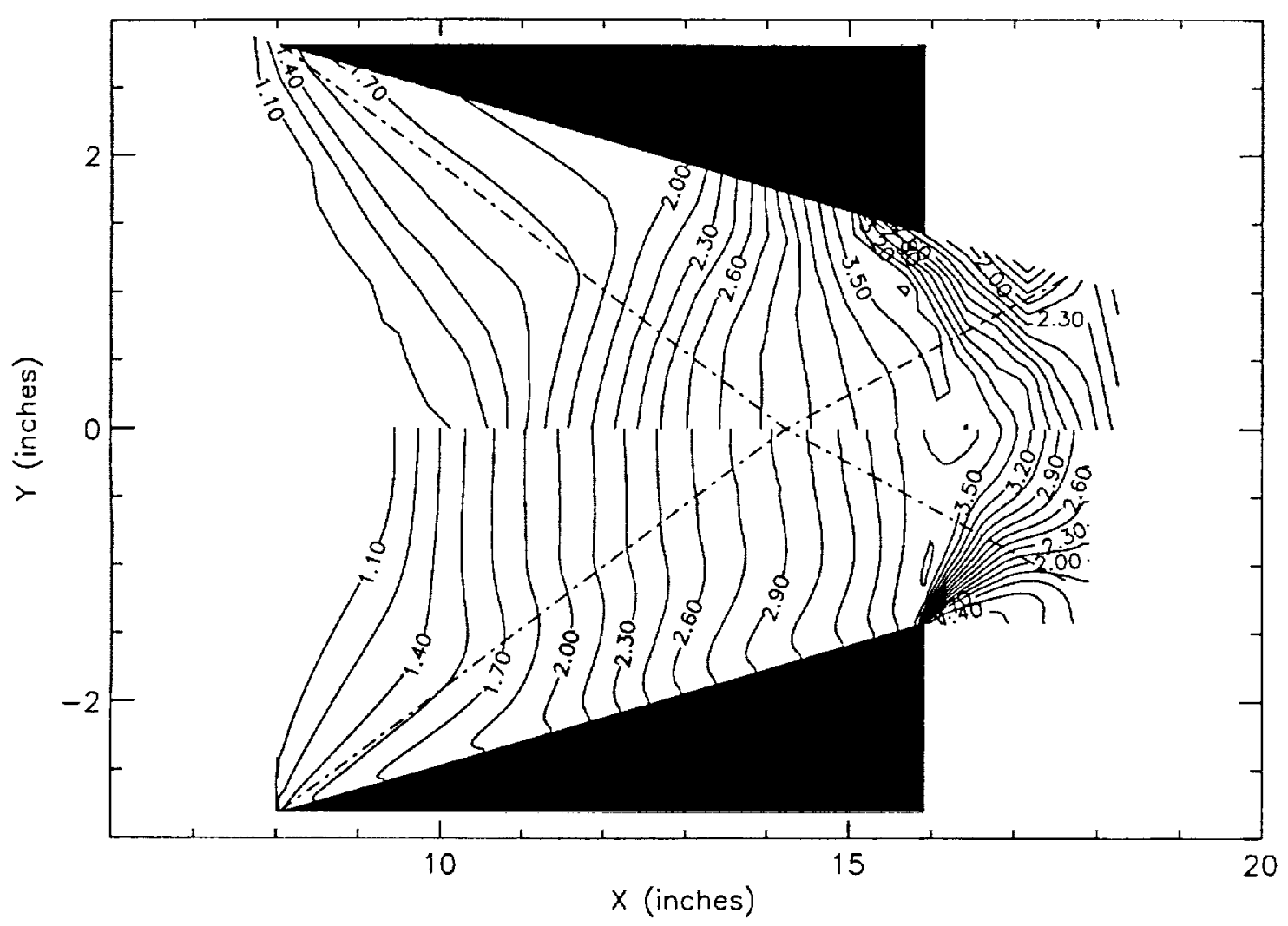

Fig. 3c Pressure Contours for 10 deg. Mach 3.5

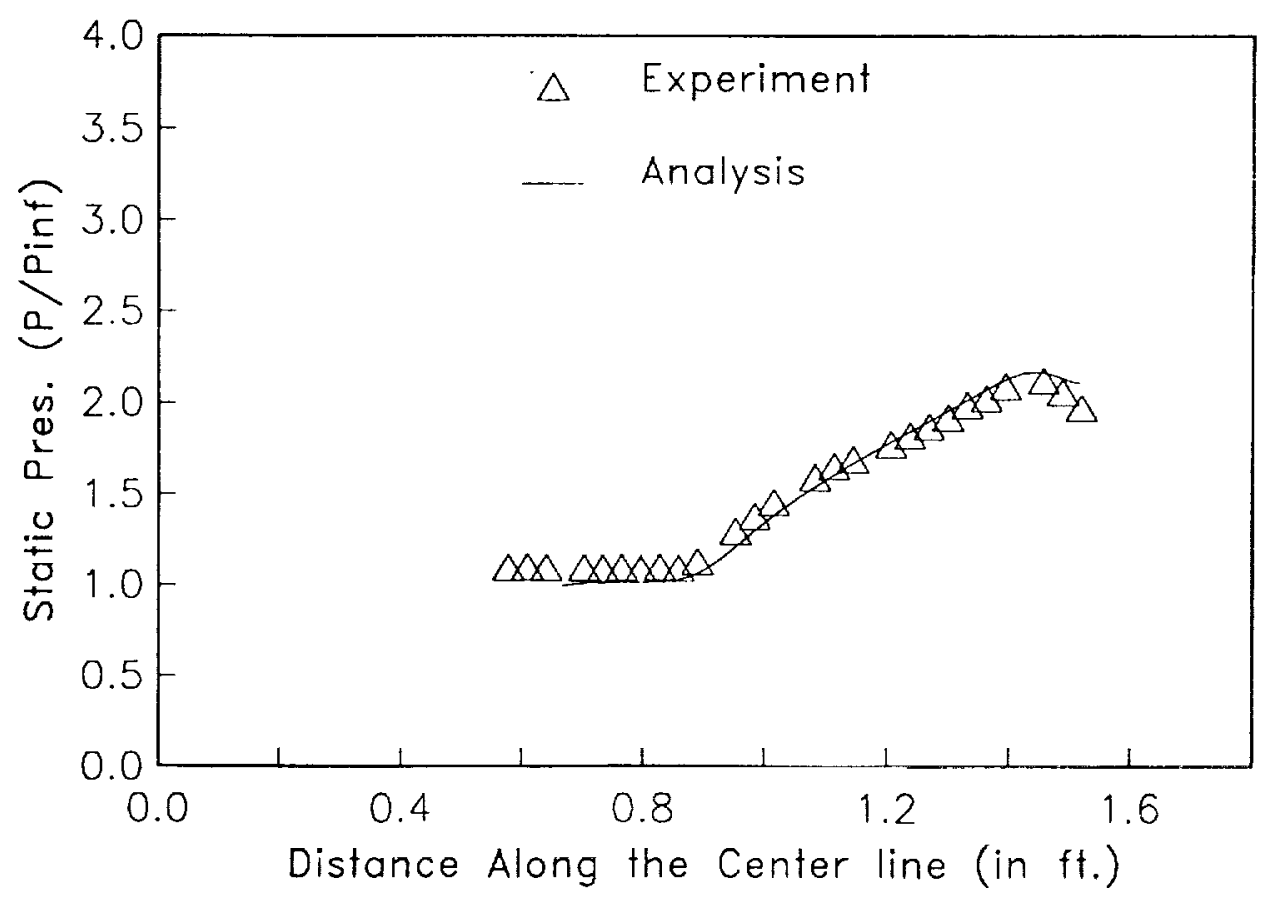

Fig. 4a Centerline Pressure Distibution for 6 deg. Mach 3.5 


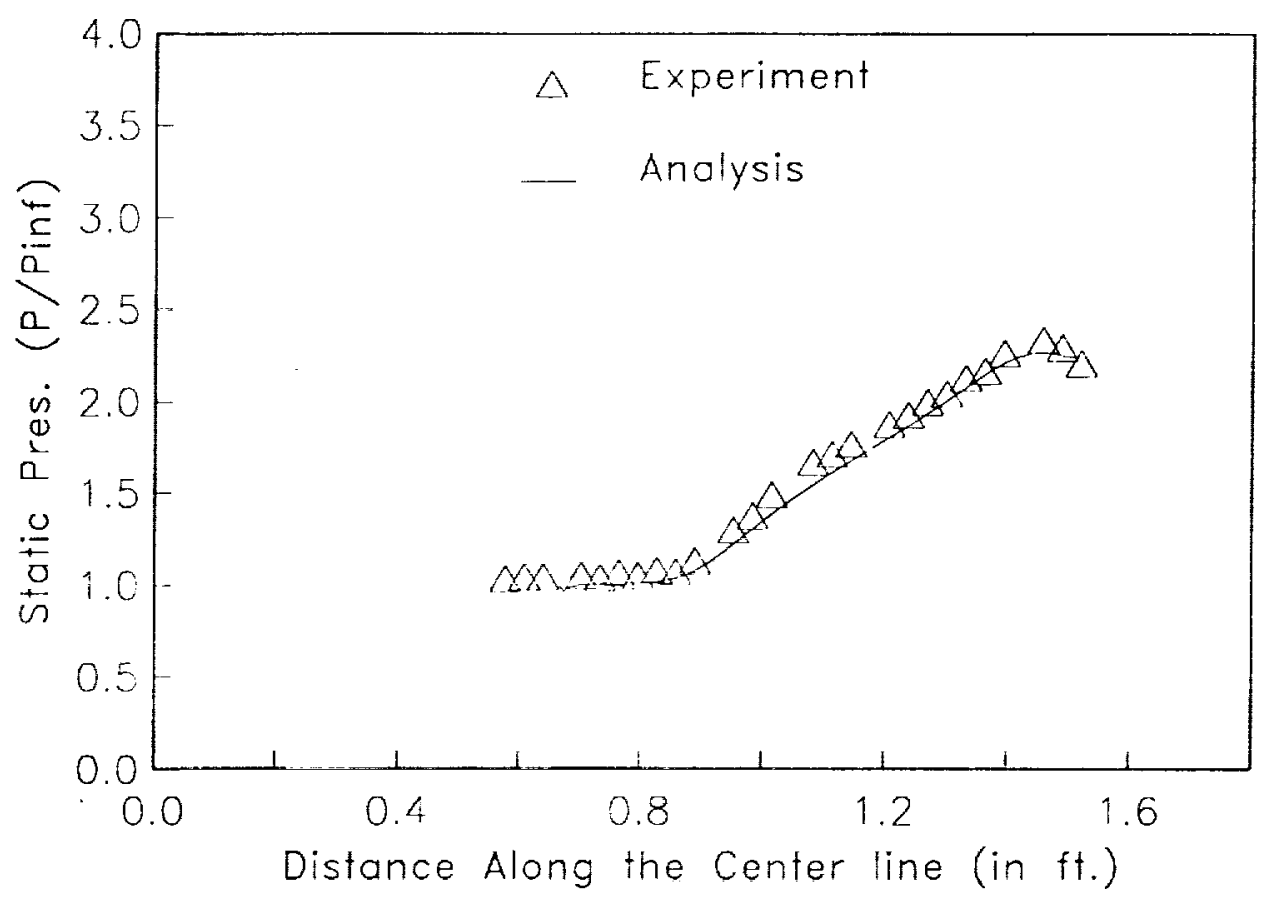

Fig. 4b Centerline Pressure Distibution for 6 deg. Mach 4.0

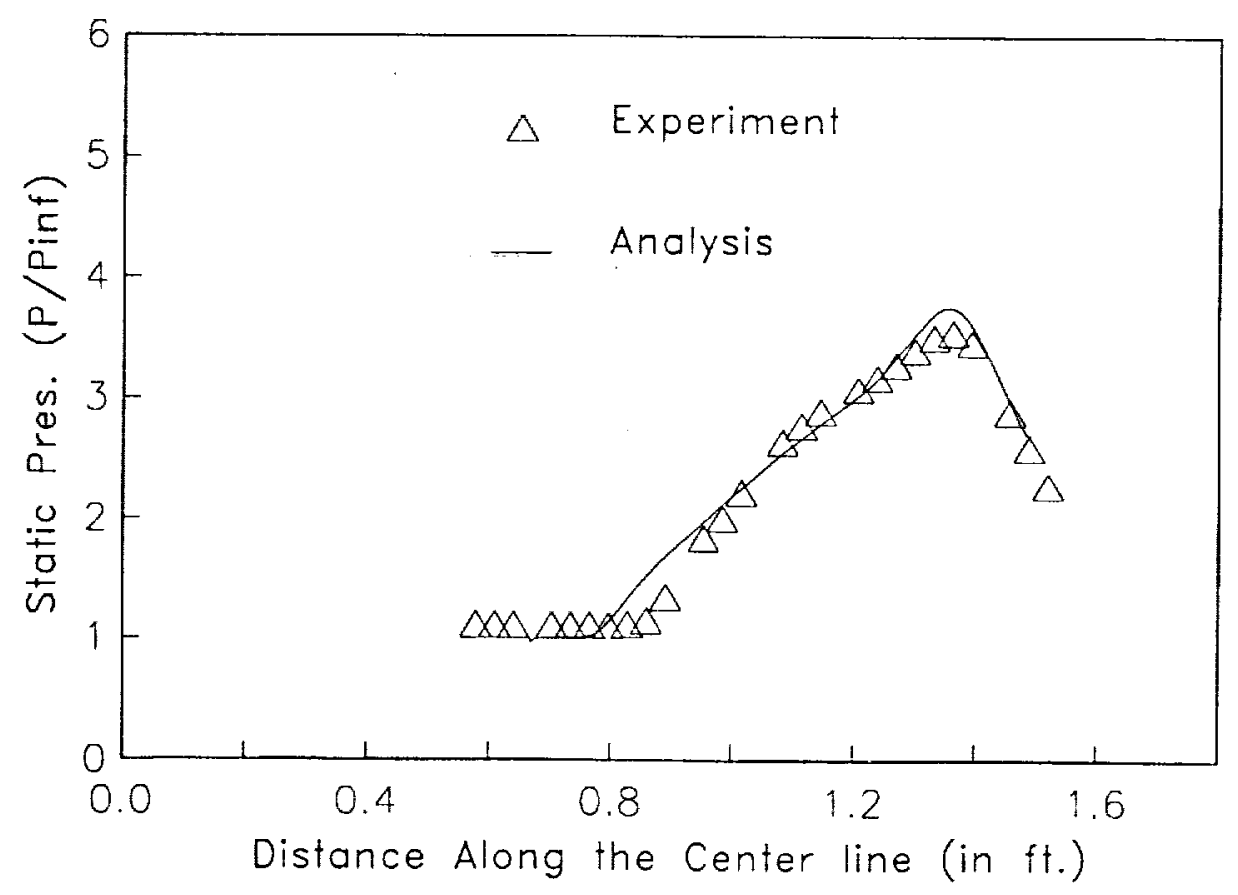

Fig. 4c Centerline Pressure Distibution for 10 deg. Mach 3.5 


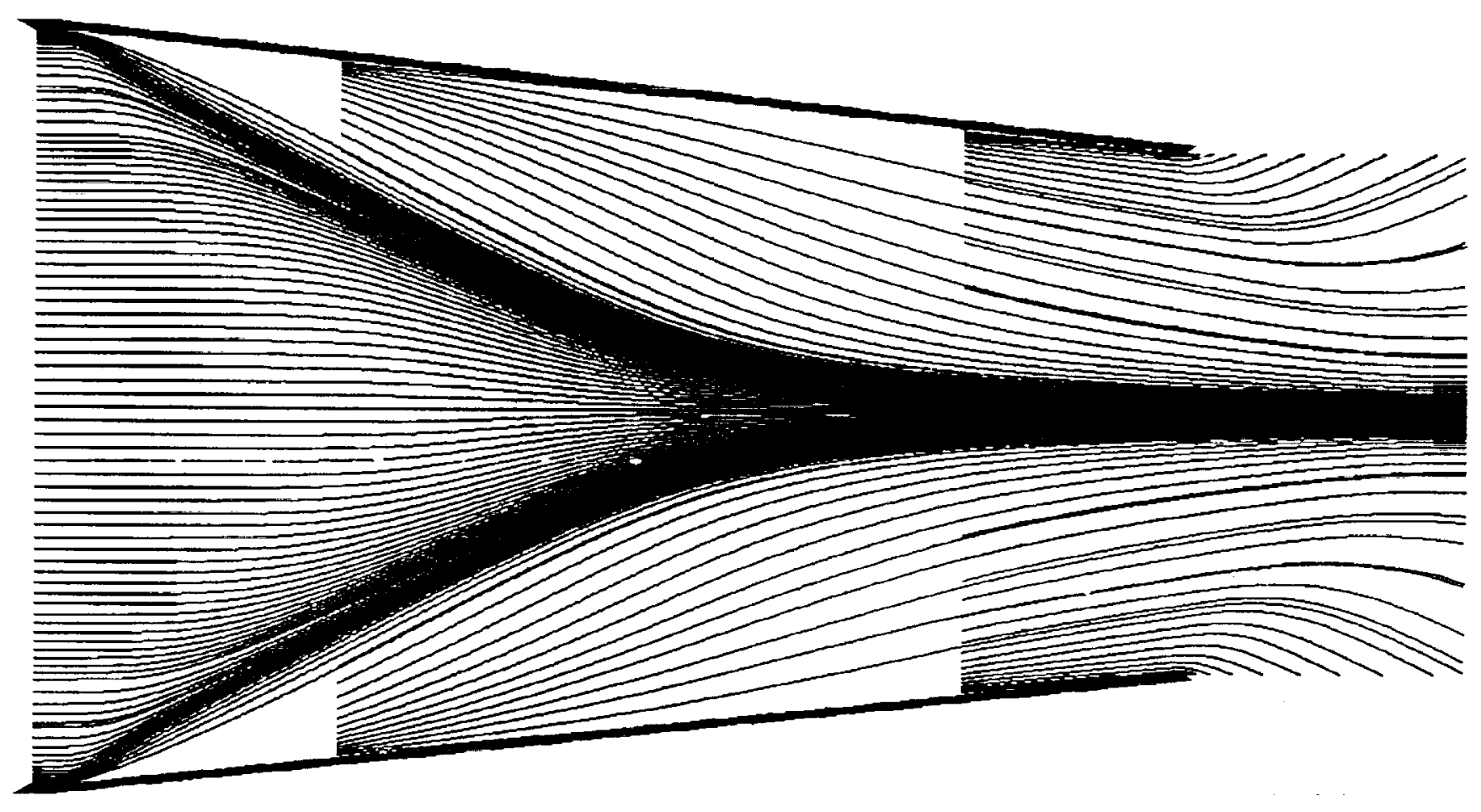

Fig. 5a Particle traces (analysis) close to tunnel wall for 6 deg. Mach 3.5

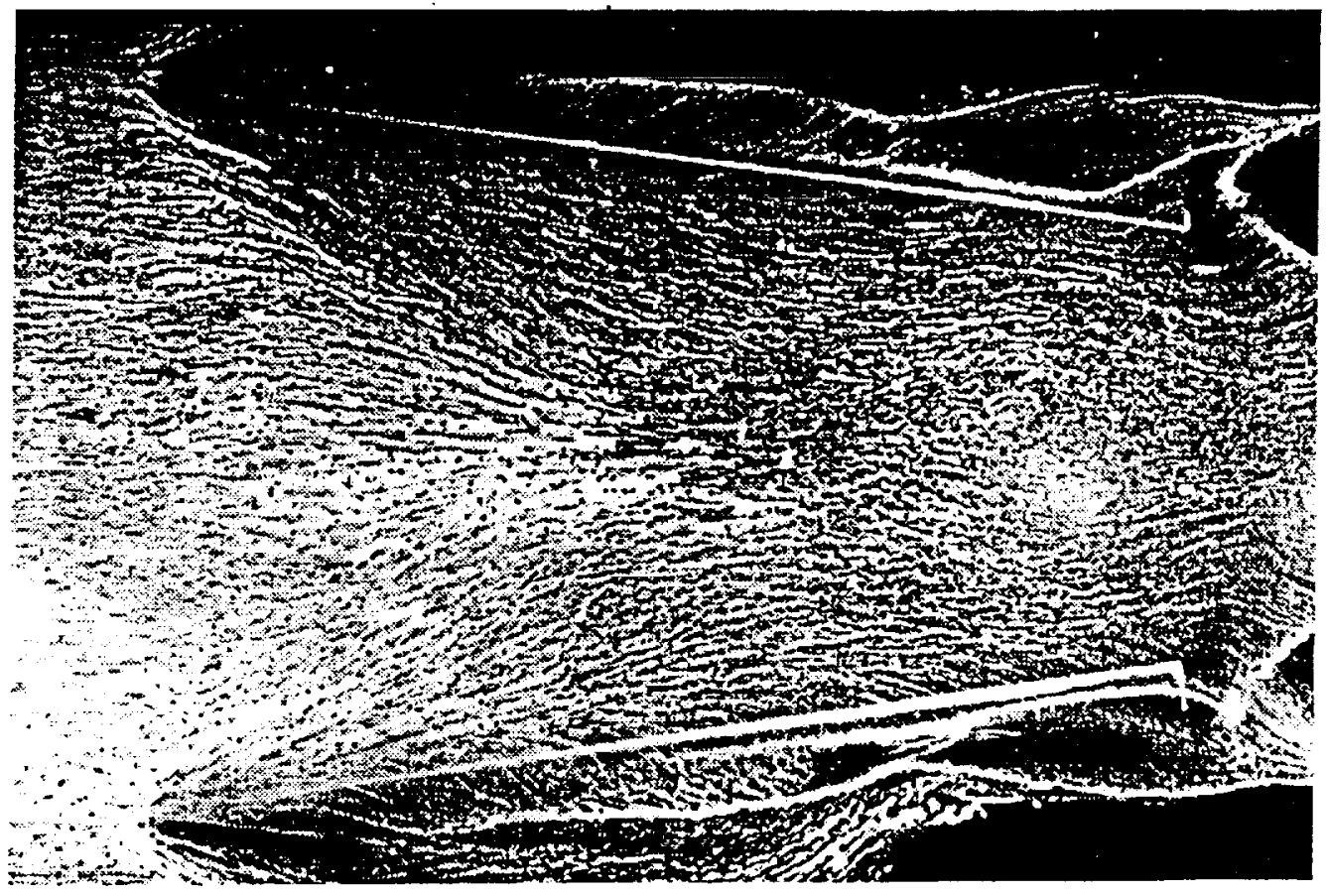

Fig. 5b Oil flow pattern (experimental) on tunnel wall for 6 deg. Mach 3.5 


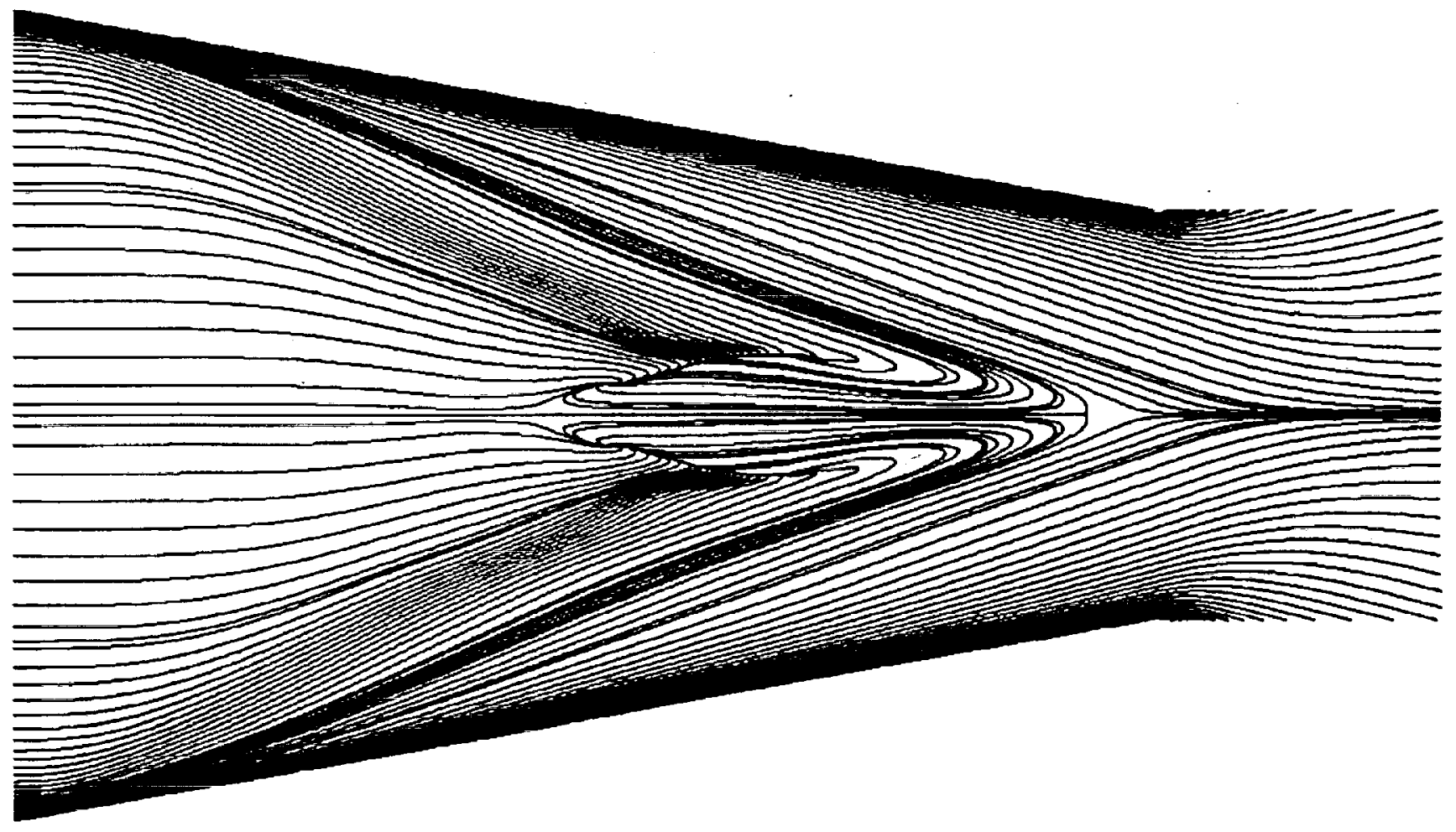

Fig. 6a Particle traces (analysis) close to tunnel wall for 10 deg. Mach 3.5

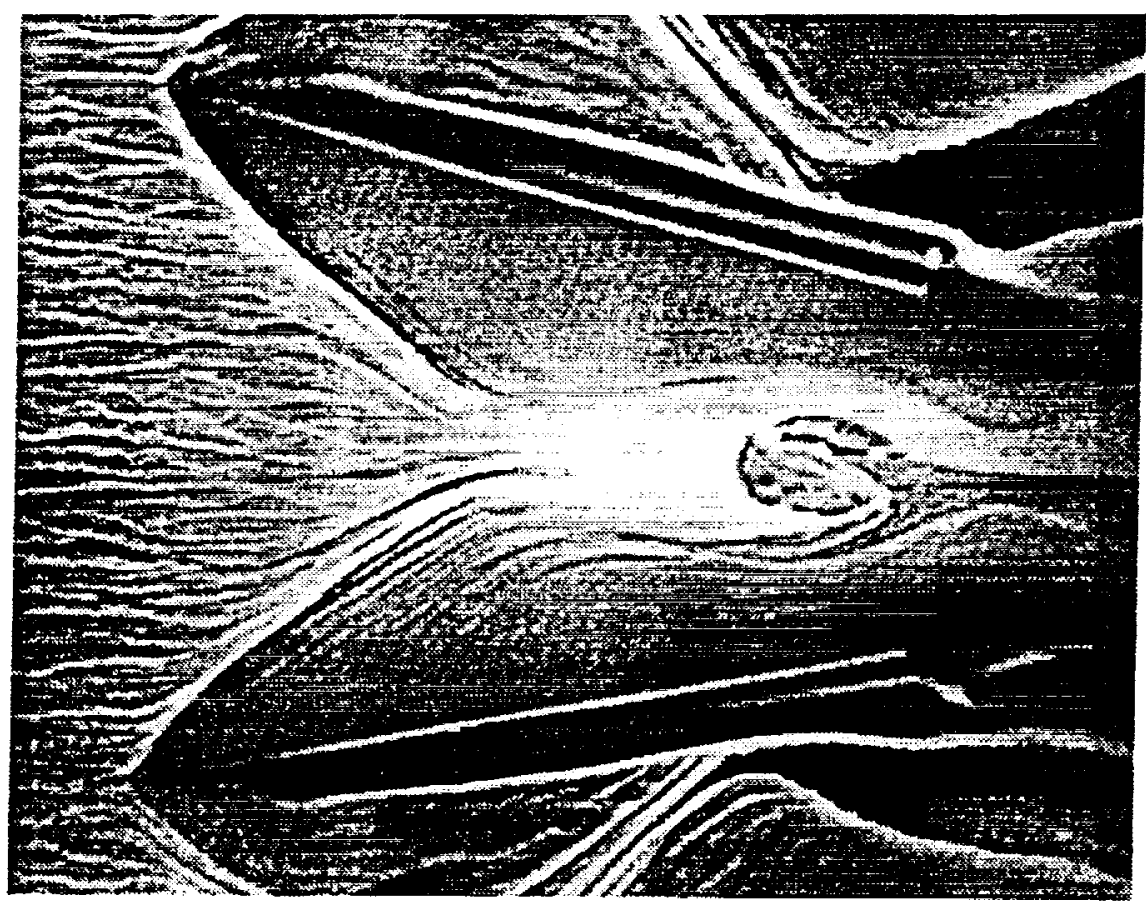

Fig. $6 b$ Oil flow pattern (experimental) on tunnel wall for 10 deg. Mach 3.5 


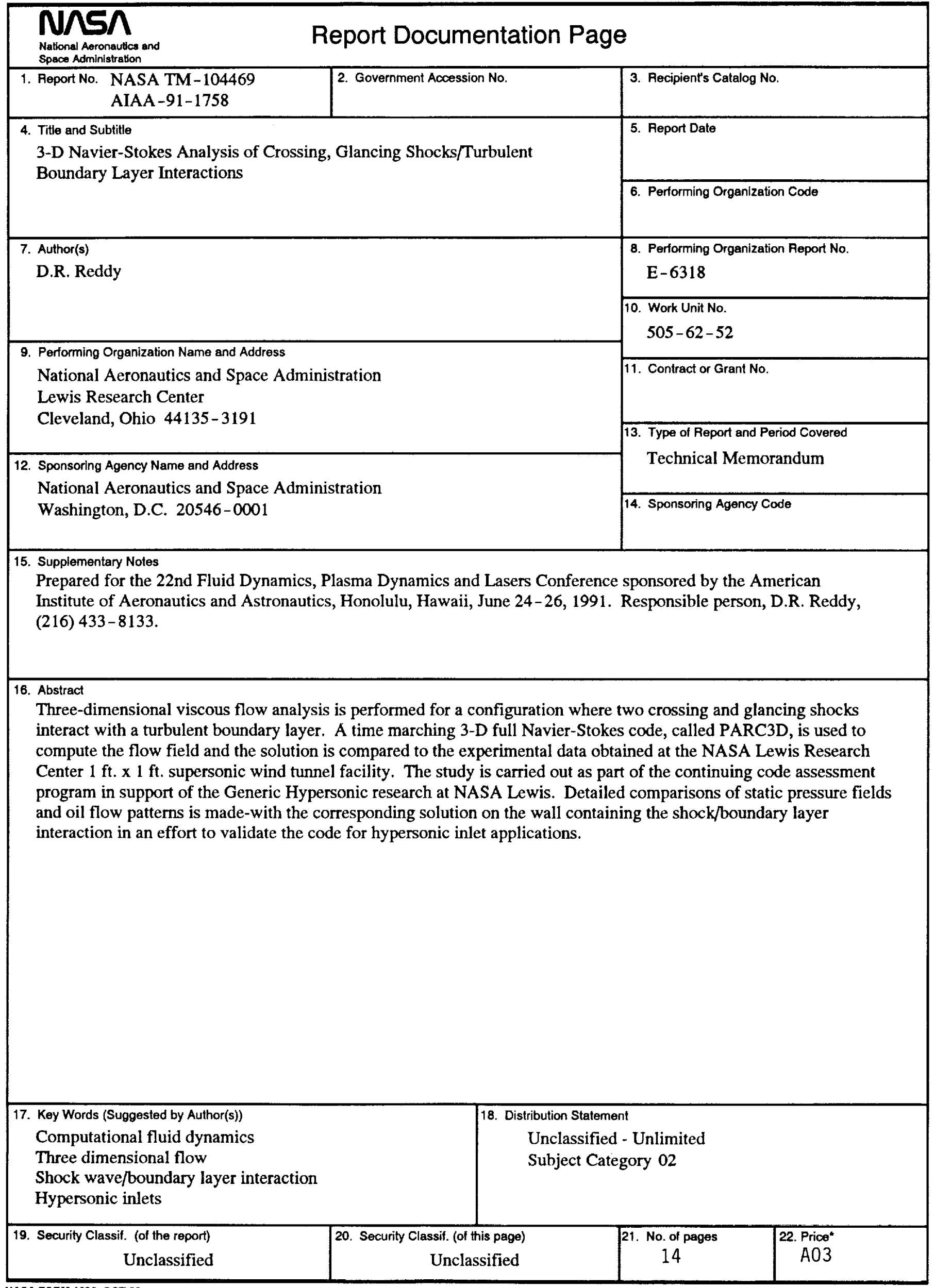


$\cdots$ 